September $11^{\text {th }}, 2002$

\title{
Overview of ATLAS B-Physics Prospects
}

\author{
Szymon Gadomski \\ LHEP, University of Bern, 3012 Bern, Switzerland \\ and Institute of Nuclear Physics, Cracow, Poland
}

For the ATLAS Collaboration

\begin{abstract}
We report on the prospects for B-physics measurements with the ATLAS detector at the LHC. The experiment will be able to collect large samples of exclusive decays containing $J / \psi$. Competitive measurement of $\sin (2 \beta)$ using $B_{d}^{0} \rightarrow J / \psi K_{s}^{0}$ and good sensitivity to new physics in $B_{s}^{0} \rightarrow J / \psi \phi^{0}$ are expected. ATLAS will also be sensitive to rare decays $B_{d, s}^{0} \rightarrow \mu^{+} \mu^{-}$and $B_{d, s}^{0} \rightarrow \mu^{+} \mu^{-} X$. Useful measurements in hadronic decay channels such as $B_{s, d}^{0} \rightarrow h^{+} h^{-}, B^{+} \rightarrow K^{+} K^{+} \pi^{-}$and $B_{s}^{0} \rightarrow D_{s}^{-} \pi^{+}$are also possible. The expected precision in these and other channels is presented.
\end{abstract}





\section{Introduction}

At the LHC $b \bar{b}$ pairs will be produced at large rates. Assuming an initial luminosity of the order of $10^{33} \mathrm{~cm}^{-2} \mathrm{~s}^{-1}$ one can expect of the order of $10^{10} \mathrm{~b} \bar{b}$ pairs per year passing the Level-1 single muon trigger of ATLAS $\left(p_{\mathrm{T}}>6 \mathrm{GeV} / \mathrm{c}\right.$ and pseudorapidity in the range of \pm 2.4 ). The LHC experiments can collect samples of exclusive B-hadron decays that will be significantly larger than at $\mathrm{B}$ factories or at the Tevatron. In the case of exclusive decays producing pairs of muons, which are relatively easy to select online, it is expected that ATLAS can have statistical precision comparable to that of the LHCb.

The ATLAS detector and trigger are described in Ref. [1]; only a very brief summary is given here. A schematic view of the detector is shown in Fig. 1. The main components of the detector are labeled in the figure. Two subdetectors of ATLAS are described in more detail these proceedings, the Electromagnetic Calorimeter in Ref. [2] and the Inner Detector in Ref. [3]. The trigger system of ATLAS is also described in these proceedings $[4]$.

The rapidity range covered by the ATLAS detector for B-physics is limited by the tracking to $|\eta|<2.5$. Tracks can be reconstructed for $p_{T}>0.5 \mathrm{GeV} / \mathrm{c}$. Muons are identified in the Muon Spectrometers for $p_{\mathrm{T}}>5 \mathrm{GeV} / \mathrm{c}$ in the barrel and for $p_{\mathrm{T}}>3 \mathrm{GeV} / \mathrm{c}$ in the end-caps. The barrel part of the hadronic Tile Calorimeter can also identify muons in the range of $3 \mathrm{GeV} / \mathrm{c}<p_{\mathrm{T}}<5 \mathrm{GeV} / \mathrm{c}$.

Results on the expected accuracies of B-physics measurements in ATLAS are documented in Ref. [1], with some updates in Ref. [5]. New results and further updates are documented in Refs. $[6,7,8]$. In this article we give a brief overview of ATLAS B-physics prospects (Section 2). We then discuss some recent developments in Section 3 and we conclude in Section 4.

\section{Expected accuracy of B-physics measurements in ATLAS}

\section{$2.1 \quad$ Decays with $J / \psi$}

A large sample of $B_{d}^{0} \rightarrow J / \psi K_{s}^{0}$ decays is expected in ATLAS. Decays of $J / \psi$ to $\mu^{+} \mu^{-}$or to $e^{+} e^{-}$will be used for the online selection of these events. The expected sample sizes are shown in Table 1. The mass spectrum obtained in simulation is shown in Fig. 2.

To perform a measurement of the CP-violating asymmetry it is necessary to know the flavour of the neutral B meson at production. Three flavour tagging algorithms have been studied in ATLAS so far. The lepton tag uses the semi-leptonic decays of the other $b$ quark (an opposite-side tag). The " $B-\pi$ " tag uses the charge of a pion produced close to the fully reconstructed B meson (same-side tag) to determine the flavour of the B meson. Finally the "jet charge" tag uses a combination of charges of particles reconstructed close to the B meson (also same-side tag).

In the case of $J / \psi \rightarrow \mu^{+} \mu^{-}$the most powerful tagging methods are the two same-side 
ones. The lepton tag has low efficiency, a semi-leptonic decay of the other $b$ is needed. For $J / \psi \rightarrow e^{+} e^{-}$however, practically all the recorded events already have a $b \rightarrow \mu X$ decay of the other $b$ quark because a muon is needed for the Level-1 trigger. For this reason the high-purity opposite-side lepton tag is the most effective tag in the $J / \psi \rightarrow e^{+} e^{-}$sample.

As the two samples predominantly use different tagging methods, the $J / \psi \rightarrow e^{+} e^{-}$ channel is useful as a check on potential systematic errors. On purely statistical grounds it is less powerful, as can be seen in Table 1. The expected combined statistical precision of ATLAS is $\delta(\sin 2 \beta)=0.010^{1}$ after $30 \mathrm{fb}^{-1}$. The statistical precision would degrade to $\delta(\sin 2 \beta)=0.015$ if only the dimuon trigger were available.

Given such high statistical precision, it will be important to control the potential systematic errors to a comparable level. Potential differences in production rates of $B_{d}^{0}$ and $\bar{B}_{d}^{0}$, which can potentially be of the order of $1 \%$ within the acceptance of ATLAS in $p_{\mathrm{T}}$ and $\eta$, may need to be corrected for. Also the asymmetries of tracking and tagging efficiencies have to be precisely controlled. Control samples of $B^{+} \rightarrow J / \psi K^{+}$and $B^{0} \rightarrow J / \psi K^{* 0}$ decays, which are expected to have no CP-violating asymmetry, can be used to measure the asymmetries of production, tracking and tagging. They can also be used to measure the dilution factor due to mistags. Large samples of the control channels are expected in ATLAS (Table 2), allowing small systematic errors on $\sin (2 \beta)$. It is therefore estimated that the precision on $\sin (2 \beta)$ will be dominated by the statistical error.

The decay $B_{s}^{0} \rightarrow J / \psi \phi^{0}$ is triggered in the same way as $B_{d}^{0} \rightarrow J / \psi K_{s}^{0}$. A signal sample of $\sim 300000$ events, with $\sim 15 \%$ background, is expected in ATLAS for an integrated luminosity of $30 \mathrm{fb}^{-1}$. The ATLAS sensitivity on the CP asymmetry in this channel is expected to be of the same order as the small asymmetry predicted in the Standard Model (SM). ATLAS would not be sensitive to the SM asymmetry, but it would be sensitive to non-SM effects that might make the asymmetry significantly larger.

Predictions for the similar decay $B_{s}^{0} \rightarrow J / \psi \eta$, which uses the electromagnetic calorimeter to reconstruct $\eta$ decays, can be found in Ref. [2].

\subsection{Hadronic decays}

The two-body hadronic decays $B_{d}^{0} \rightarrow \pi^{+} \pi^{-}$and $B_{s}^{0} \rightarrow K^{+} K^{-}$are expected to provide measurements of $\mathrm{CP}$ asymmetries related to the angles $\alpha$ and $\gamma$. As ATLAS does not have event-by-event particle identification, the signals from all the significant two-body decays of b-hadrons $\left(B_{d}^{0} \rightarrow \pi^{+} \pi^{-}, B_{d}^{0} \rightarrow K^{+} \pi^{-}, B_{s}^{0} \rightarrow \pi^{+} K^{-}, B_{s}^{0} \rightarrow K^{+} K^{-}, \Lambda_{b} \rightarrow p \pi^{-}\right.$ or $\left.p K^{-}\right)$will overlap, as shown in Fig. 3 .

The numbers of events in the peak shown in Fig. 3 are of the order of several thousands. The number of recorded $B_{d}^{0} \rightarrow \pi^{+} \pi^{-}$decays is 9500, for instance. At this large event numbers the $\mathrm{K} / \pi$ separation provided by the Inner Detector, which is of the order of $0.8 \cdot \sigma[3]$, is useful. A study was made for ATLAS with an event-by-event likelihood fit using all the available information: measured proper time, reconstructed mass, a $\mathrm{K} / \pi$ separation variable, $B_{d}^{0}$ and $B_{s}^{0}$ oscillations, as well as the time-dependence of the CP

\footnotetext{
${ }^{1}$ In this article we refer to the parameterization of the CKM matrix that is usually used for the experiments at hadron colliders. The parameterization is described in Ref. [5].
} 
asymmetry. The obtained resolution on the amplitudes of the CP asymmetry is around 0.1 , in both decay channels and for both the direct and mixing-induced asymmetries. With this precision a resolution of the order of a few degrees on the angles $\alpha$ and $\gamma$ might be possible ${ }^{2}$.

The fully hadronic decays $B_{s}^{0} \rightarrow D_{s}^{-} \pi^{+}$and $B_{s}^{0} \rightarrow D_{s}^{-} a_{1}^{+}$, followed by $D_{s}^{-} \rightarrow \phi \pi^{-}$ can be used to measure the $B_{s}^{0}$ mixing oscillation frequency $\Delta\left(m_{s}\right)$. The sample of fully reconstructed $B_{s}^{0}$ decays is expected to be $\sim 3300$ after $10 \mathrm{fb}^{-1}[7]$. The analysis following ATLAS simulation used an amplitude-fit technique to estimate the ATLAS sensitivity to $x_{s}$. In Fig. 4 the points with error bars show one simulated experiment with the ATLAS statistics and with the ATLAS resolution on the proper time of the $B_{s}^{0}$ decay. By repeating such simulated experiments many times one obtains the sensitivities of ATLAS expected after $10 \mathrm{fb}^{-1}$. A measurement of $\Delta m_{s}$ with significance above $5 \sigma$ is possible for $\Delta m_{s}<22.5 \mathrm{ps}^{-1}$. A $95 \%$ confidence exclusion limit on can be set on average up to $\Delta m_{s}=34.5 \mathrm{ps}^{-1}$.

The decay $B^{+} \rightarrow K^{+} K^{+} \pi^{-}$was studied recently in ATLAS [8]. This decay is highly suppressed in the SM where the expected branching fraction is $\sim 10^{-11}$. Some extensions of the SM predict branching ratios that are several orders of magnitude larger. Such signals would be visible (or could be excluded) by ATLAS. It is expected that with an integrated luminosity of $30 \mathrm{fb}^{-1}$ ATLAS would be able to set a $95 \%$ C.L. exclusion limit on the brancing ratio $\mathcal{B}\left(B^{+} \rightarrow K^{+} K^{+} \pi^{-}\right)$at the level of $3.5 \cdot 10^{-7}$.

\subsection{Rare decays with dimuons}

The decays $B_{s}^{0} \rightarrow \mu^{+} \mu^{-}$and $B_{d}^{0} \rightarrow \mu^{+} \mu^{-}$have very small SM branching ratios but they can be selected online by ATLAS even at highest LHC luminosities. Looking at these decays offers a good possibility to search for evidence of new physics. As an example, after three nominal years $\left(10^{7} \mathrm{~s}\right)$ of the LHC running at $10^{33} \mathrm{~cm}^{-2} \mathrm{~s}^{-1}$, followed by one year of LHC running at $10^{34} \mathrm{~cm}^{-2} \mathrm{~s}^{-1}$, the decay $B_{s}^{0} \rightarrow \mu^{+} \mu^{-}$would be visible in ATLAS at the level of $4.7 \sigma$, assuming the SM branching ratio of $4.9 \cdot 10^{-9}$.

Another class of interesting rare decays with dimuons are decays of the type $B_{d, s}^{0} \rightarrow$ $X \mu^{+} \mu^{-}$, such as $B_{d}^{0} \rightarrow K^{* 0} \mu^{+} \mu^{-}, B_{d}^{0} \rightarrow \rho^{0} \mu^{+} \mu^{-}$and $B_{s}^{0} \rightarrow \phi^{0} \mu^{+} \mu^{-}$. ATLAS will be able to measure branching ratios for these decays, which provide a handle on CKM matrix elements. A study done for ATLAS also indicates that an asymmetry in $B_{d}^{0} \rightarrow$ $K^{* 0} \mu^{+} \mu^{-}$could be used to obtain evidence of some extensions of the Standard Model. The asymmetry, called a forward-backward asymmetry $A_{F B}$, is relative to the angle between the $\mu^{+}$and the $B$-meson momentum in the rest frame of the $\mu^{+} \mu^{-}$pair. The potential of ATLAS for this analysis is presented in Ref [1].

\footnotetext{
${ }^{2}$ The accuracy depends on how well the penguin contribution is known. It also depends on the values of $\alpha$ and $\gamma$. More information is given in Ref. [5]
} 


\subsection{Measurements of $b$-production}

Measurements of $b$-Production offer a probe of perturbative QCD. The production must also be understood in order to estimate $b \bar{b} b \bar{b}$-production, which is a background to Higgs decays.

Measurements of absolute and differential cross-sections can be made by ATLAS using the exclusive decays mentioned above, as well as using more inclusive samples such as $b \rightarrow J / \psi X$ decays. One can also use an even more inclusive technique of tagging b-jets using purely the impact-parameter information, not requiring $J / \psi$. The area in $\left(p_{T}, \eta\right)$ space covered by ATLAS for b-production measurements is complementary to the coverage of LHCb. However there is sufficient overlap to allow for a cross-check and for a common normalization of the results.

Measurement of the angular correlation between the two quarks in $b \bar{b}$ events is a way to check next-to-leading order or higher contributions to the $b$-quark production crosssection. The azimuthal separation $\Delta \phi_{b \bar{b}}$ is an interesting variable from this point of view. Traditionally such correlations are measured in dimuon events, assuming that both muons are the product of semi-leptonic decays of $b$ hadrons. Because of high background such analyses often need to exclude the region of low $\Delta \phi_{b \bar{b}}$, where there is most interest. In ATLAS, because of high statistics, it will be possible to measure $\Delta \phi_{b \bar{b}}$ using events containing a $J / \psi$. This technique reduces the background and allows one to approach the regions of small $\Delta \phi_{b \bar{b}}$. More details can be found in Ref. [9].

\section{Recent developments}

It is expected that the high-level trigger system of ATLAS may initially need to function with a reduced data-movement and processing capacity due to financial constraints. In another recent development the target initial luminosity of the LHC machine was doubled to $2 \cdot 10^{33} \mathrm{~cm}^{-2} \mathrm{~s}^{-1}$. Both factors would make the online selection of B-decays more difficult. Some algorithms needed for the online selection of B events are demanding on CPU power.

In spite of the above considerations ATLAS needs to be prepared to take maximum advantage of the available luminosity in the first years of running. The target of 2 . $10^{33} \mathrm{~cm}^{-2} \mathrm{~s}^{-1}$ might not be achieved immediately.

Furthermore the luminosity is not constant during a coast. A drop by factors of typically two or three is expected during a typical collision period corresponding to one fill of the LHC. It is expected that ATLAS trigger menus will be modified as a function of the instantaneous luminosity. The dimuon trigger, which is the least CPU demanding, may be the only B-physics trigger used at $2 \cdot 10^{33} \mathrm{~cm}^{-2} \mathrm{~s}^{-1}$. The selection of hadronic channels in the ATLAS high-level triggers might only be switched on at lower luminosities. 


\section{Conclusions}

ATLAS can make several useful measurements in the B-physics sector. It will have a sensitivity on $\sin 2 \beta$ comparable to that of $\mathrm{LHCb}$ and it can contribute a significant measurement of $B_{s}^{0}$ mixing. Sensitivity in several other channels, e.g. $B_{s}^{0} \rightarrow J / \psi \phi^{0}$, $B_{s}^{0} \rightarrow \mu^{+} \mu^{-}$and $B_{d}^{0} \rightarrow K^{* 0} \mu^{+} \mu^{-}$, which can be selected online with relative ease thanks to dimuons, is sufficient to allow precise tests of the Standard Model and potentially evidence of new physics.

\section{References}

[1] ATLAS Detector and Physics Performance Technical Design Report, CERN/LHCC/99-14 and 99-15, 25 May 1999.

[2] Sebastien Viret, these proceedings.

[3] Eduardo Ross, these proceedings.

[4] John Baines, these proceedings.

[5] G. Altarelli, M. Mangano (Eds.) Proceedings of the workshop on the Standard Model physics (and more) at the LHC, CERN 2000-004.

[6] C. Driouichi, P. Eerola, M. Melcher, F. Ohlsson-Malek, S. Viret, 'Observation potential of the decays $B_{s, d}^{0} \rightarrow J / \psi \eta$ ', EPJdirect CN2, 1-13 (2002).

[7] B. Epp, V.M. Ghete, A. Nairz, 'Prospects for the measurement of $B_{s}^{0}$ oscillations with the ATLAS detector at LHC', EPJdirect CN3, 1-23 (2002).

[8] J. Damet, P. Eerola, A. Manara and S.E.M. Nooij, 'Searching for physics beyond the Standard Model in the decay $B^{+} \rightarrow K^{+} K^{+} \pi^{-}$, EPJdirect C7, 1-9, (2001).

[9] Simon Robins, 'b-Production Measurements with ATLAS', Nucl. Instrum. Methods A 462 (2001) 184-188. 


\begin{tabular}{|l|c|c|}
\hline Decay of $J / \psi$ & $J / \psi \rightarrow \mu^{+} \mu^{-}$ & $J / \psi \rightarrow e^{+} e^{-}$ \\
\hline$N\left(B_{d}^{0} \rightarrow J / \psi K_{s}^{0}\right)$ & 160000 & 4800 \\
signal/background & 30 & 15 \\
$\delta(\sin 2 \beta)$ (stat.) & 0.022 & 0.031 \\
\hline
\end{tabular}

Table 1: Expected numbers of reconstructed and tagged $B_{d}^{0} \rightarrow J / \psi K_{s}^{0}$ decays and the corresponding statistical precision on $\sin (2 \beta)$ for an integrated luminosity of $10 \mathrm{fb}^{-1}$ in ATLAS.

\begin{tabular}{|l|c|c|}
\hline Flavour tag & Lepton tag & B- $\pi$ tag \\
\hline$B^{+} \rightarrow J / \psi K^{+}$ & 41000 & 690000 \\
$B^{0} \rightarrow J / \psi K^{* 0}$ & 21000 & 360000 \\
\hline
\end{tabular}

Table 2: Expected numbers of reconstructed and tagged events in control samples for an integrated luminosity of $10 \mathrm{fb}^{-1}$ in ATLAS. 


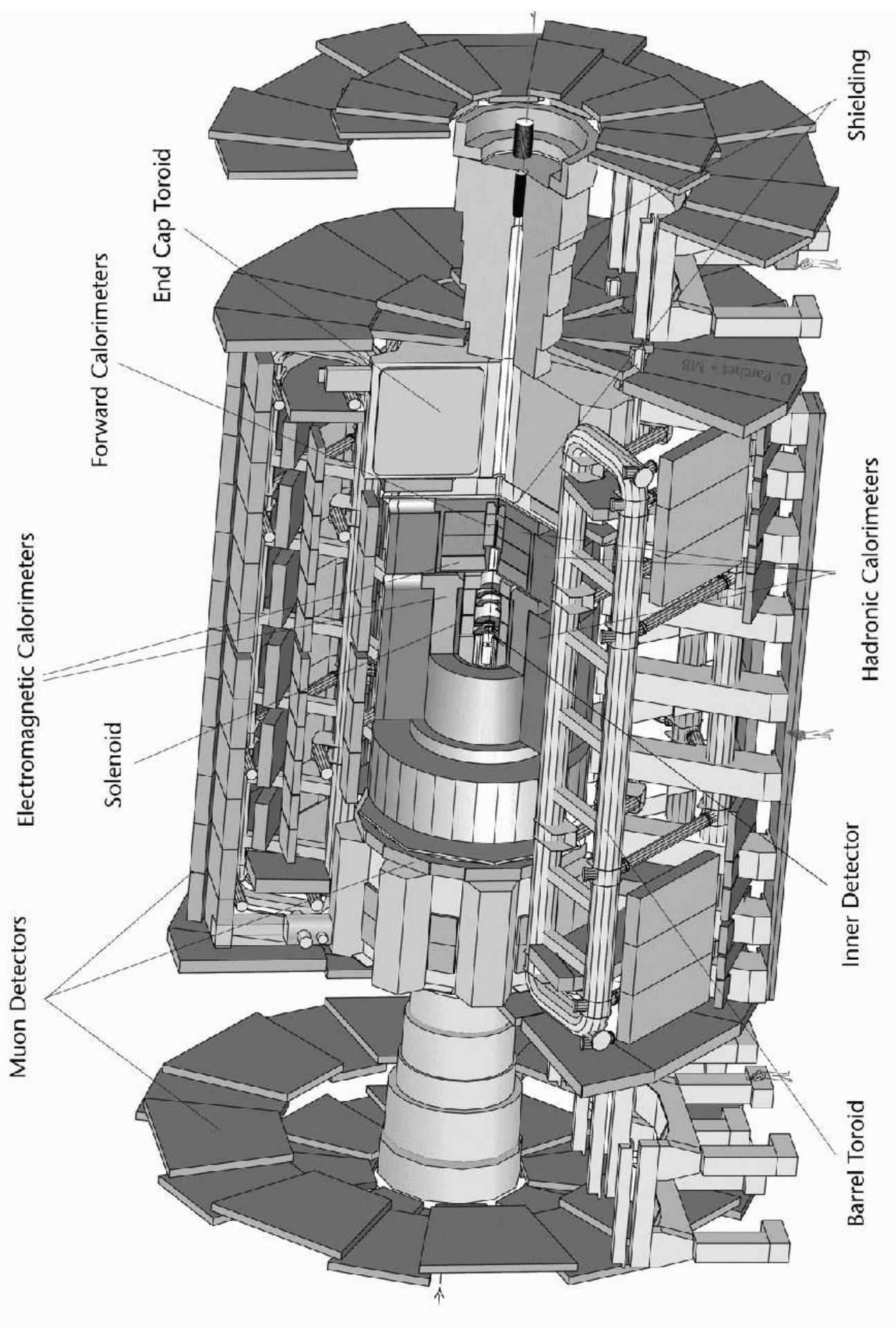

Figure 1: A view of the complete ATLAS detector. 


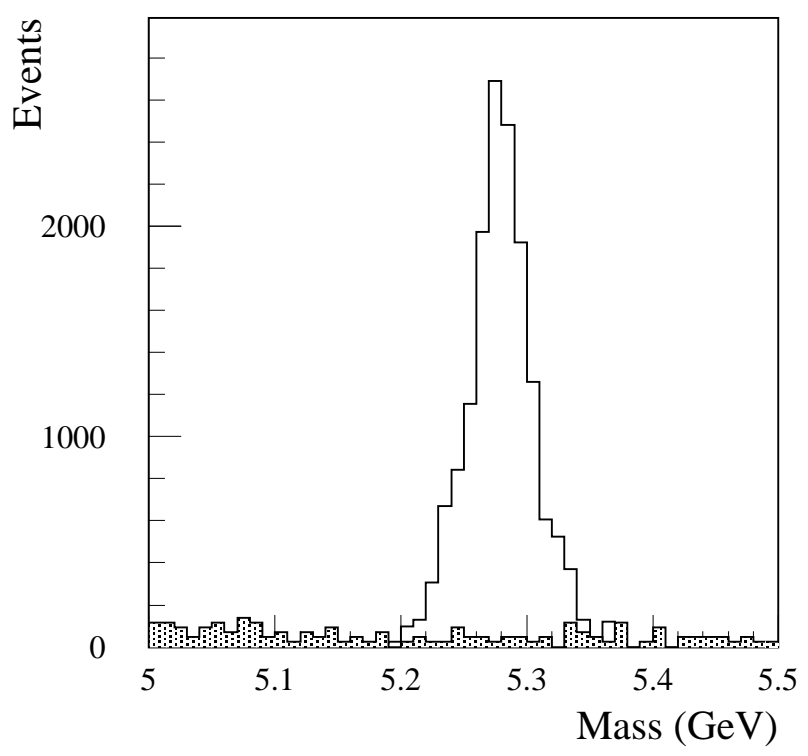

Figure 2: Expected mass spectrum of $J / \psi K_{s}^{0}$ candidates with signal (white) and background (hatched) after $30 \mathrm{fb}^{-1}$.

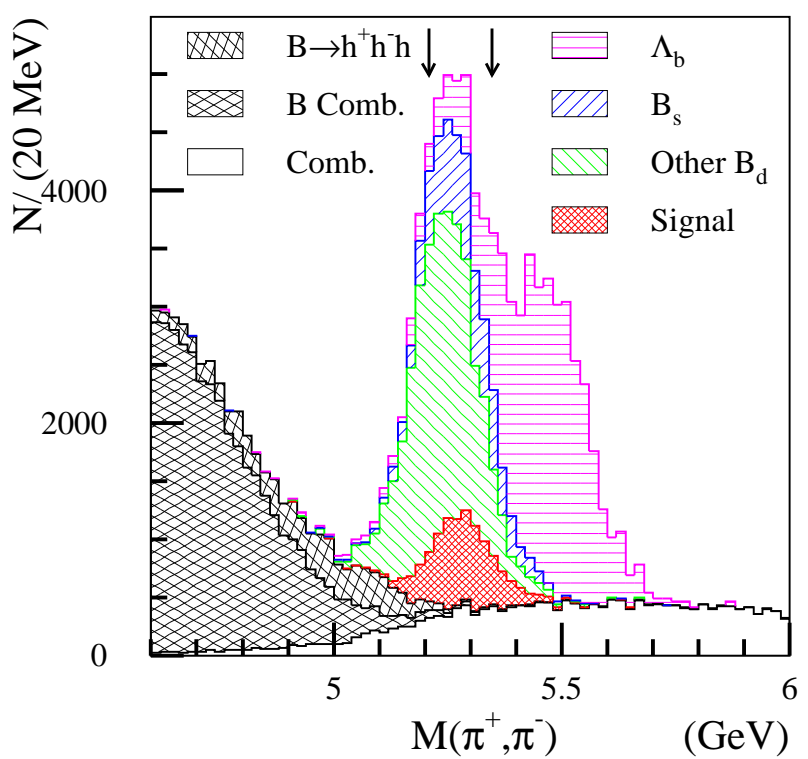

Figure 3: Expected mass spectrum of $B \rightarrow \pi^{+} \pi^{-}$candidates. The "B Comb" distribution contains the entries with two tracks coming from the same B hadron that are not included in the other distributions. The "Comb" distribution contains all other combinatorial background. 


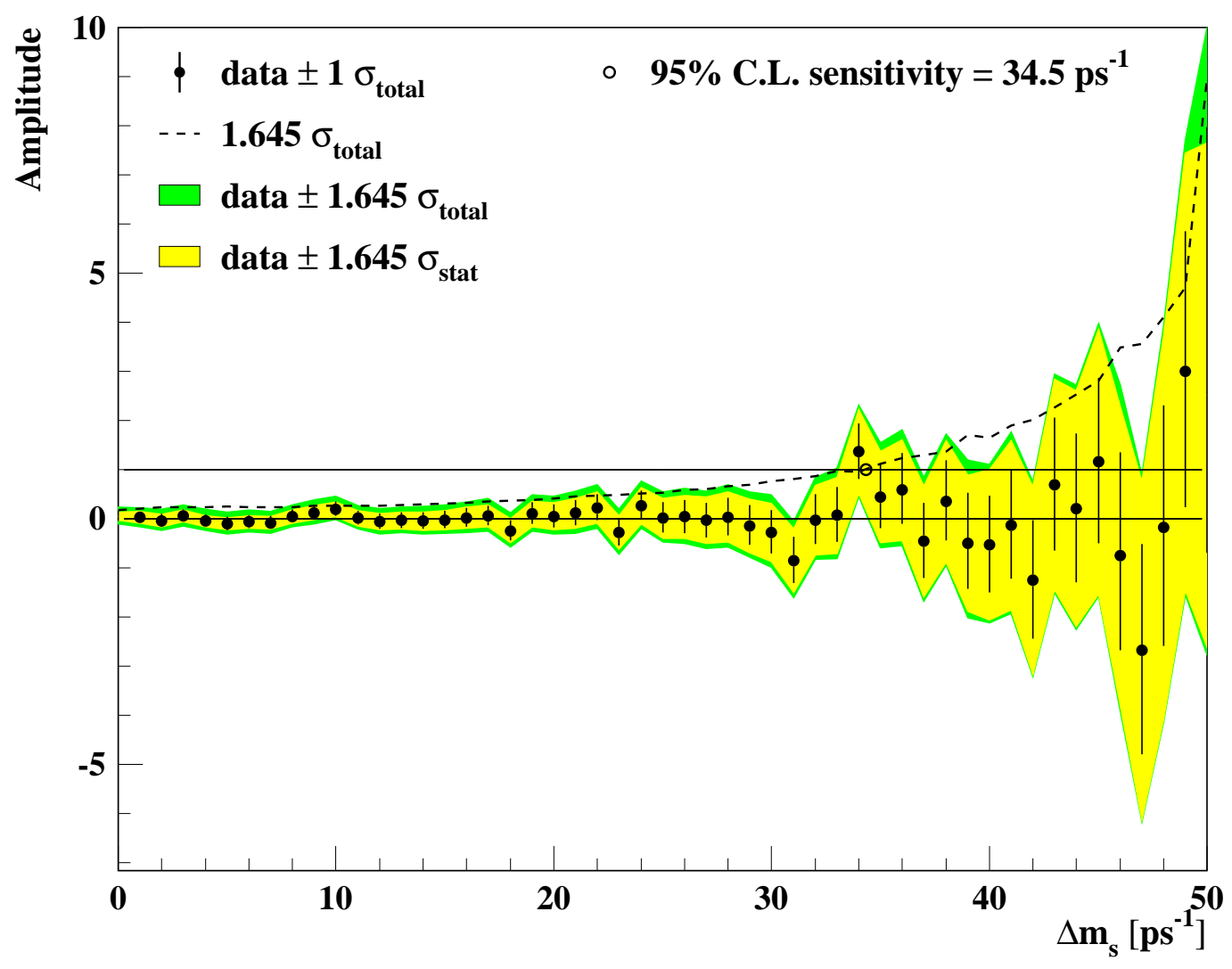

Figure 4: Results of an amplitude fit with simulated ATLAS candidates for exclusive decays of $B_{s}^{0}$ after $10 \mathrm{fb}^{-1}$ for the $\Delta m_{s}=\infty$. The "data" points show the results of an experiment with the statistics and the resolutions of ATLAS done once. Repeating such simulation gives an average fitted amplitude consistent with 0 and the values of $\sigma_{\text {stat }}$ (statistical errors only) and $\sigma_{\text {total }}$ (with an estimate of systematic errors added) increasing with $\Delta m_{s}$. The ATLAS sensitivity for setting the $95 \%$ C.L. exclusion limit is the point where $1.645 \cdot \sigma_{\text {total }}=1$. The $5 \cdot \sigma$ discovery limit is the point where $5 \cdot \sigma=1$. 